\title{
Gestión Estratégica de Recursos Humanos como Antecedente del Balanced Scorecard
}

\section{Strategic Management of Human Resources as Antecedents to the Balanced Scorecard}

\author{
Joan Boada-Grau \\ Universitat Rovira i Virgili
}

\author{
Carme Gil-Ripoll \\ Escuela de Alta dirección y Administración \\ (EADA)
}

\begin{abstract}
Resumen. En el presente estudio empírico la Gestión Estratégica de Recursos Humanos se erige como antecedente del modelo Balanced Scorecard. El objetivo de dicho estudio es examinar la relación entre la Gestión Estratégica de Recursos Humanos implementada en las organizaciones con las tres perspectivas (clientes, finanzas y procesos) del Balanced Scorecard. En la muestra han participado 222 directivos a los cuales se les aplicaron varias pruebas auto-administradas que contemplaban aspectos como: los datos demográficos del entrevistado y de la empresa, la escala BSC-14 y la escala GERH-53. Los resultados y las conclusiones obtenidas hacen referencia a que la Gestión Estratégica de Recursos Humanos predice los parámetros del Balanced Scorecard.

Palabras clave: gestión estratégica de recursos humanos, Balanced Scorecard, directivos, predicción.
\end{abstract}

\begin{abstract}
In this empirical study, Strategic Human Resources Management becomes a precursor to the Balanced Scorecard. The aim of the study is to examine the relationship between Strategic Human Resources Management in organisations implemented using the three perspectives (customer, financial and process) of the Balanced Scorecard. Two hundred and twenty-two directors took part in this sample, all of whom were asked to do several self-administered tests covering aspects like: demographic data of the interviewee and the company, the BSC-14 scale and the SHRM-53 scale. The results obtained and conclusions drawn suggest that Strategic Human Resources Management predicts the parameters of the Balanced Scorecard.

Key words: strategic human resource management, Balanced Scorecard, managers, prediction.
\end{abstract}

Las organizaciones, en el siglo XXI, están inmersas en una profunda transformación. El entorno de la era de la información y de las nuevas tecnologías es complejo, dinámico y turbulento exigiendo, tanto a las organizaciones industriales como a las de servicios, diseñar nuevas estrategias para obtener un nivel de sostenibilidad que les permita abordar adecuadamente el futuro con éxito. Durante los años noventa del pasado siglo, Kaplan y Norton (1992 y 1996) introdujeron el modelo del Balanced Scorecard (BSC, en adelante) que permite planificar y evaluar las organizaciones. Pasada más de una década este modelo se ha hecho cada vez más presente a nivel internacional, entre otros ejemplos, en USA (Kaplan y Norton, 2007; Klein y Nicholson, 2006), en Europa (Braam, Benders y Heusinkveld, 2007; Schreiber, 2007; Wisniewski y Olafsson, 2004), en Asia (Keramati, Mehrabi y

Joan Boada-Grau, Departament de Psicología, Universitat Rovira i Virgili; Carme Gil-Ripoll, Centro de Investigación en Evaluación y Medida de la Conducta (CRAMC), Escuela de Alta Dirección y Administración (EADA). La correspondencia sobre este artículo deberá enviarse a Joan Boada-Grau, Departament de Psicologia, Universitat Rovira i Virgili, Carretera de Valls, s/n, 43007 Tarragona. E-mail: joan.boada@urv.cat
Mousakhani, 2008; Rhodes, Walsh y Lok, 2008) y en Oceanía (Greatbanks y Tapp, 2007).

El modelo del BSC de Kaplan y Norton (2001, 2005a, 2005b, 2006) incide en cuatro perspectivas estratégicas de las organizaciones. La primera de ellas es la financiera que permite proporcionar rendimientos superiores a la inversión desembolsada. Esta perspectiva permite hacer explícitos los objetivos financieros de la organización, adaptándolos a las distintas unidades de negocio, en las diferentes fases de su crecimiento y ciclo de vida. El BSC permite establecer ratios de medida de una correcta estrategia financiera como (1) el crecimiento y la diversificación de los ingresos (como por ejemplo: nuevos productos y servicios, aplicaciones, clientes y mercados, sinergias y alianzas, estrategias de precios, etc.), (2) la reducción de coste y mejora de la productividad (como por ejemplo: aumento de los ingresos, reducción de costes unitarios, reducción de los gastos de explotación, etc.) y (3) utilización de los activos y estrategia de inversión (como por ejemplo, el ciclo de caja o la mejora de la utilización de los activos).

Otra perspectiva del BSC es la de los clientes. Las modernas organizaciones han de identificar los segmentos de mercado, los clientes existentes y poten- 
ciales, y posteriormente seleccionar en los nichos que desean competir. En este sentido, deberían de tener un conjunto de indicadores centrales tanto del cliente (incremento, adquisición, satisfacción y rentabilidad) y de la cuota de mercado, como del producto y/o servicio, también deben valorarse los atributos intrínsecos y sus expectativas, la imagen y el presitigio de los mismos.

La tercera perspectiva del BSC incluye los procesos internos (o workflows) de la organización. Éstos se identifican con el fin de mejorarlos y tender a la excelencia. Así se satisfacen las demandas y necesidades de los clientes tanto internos como externos. En este sentido, se deberá reconocer la cadena de valor del proceso operativo interno desde la recepción del pedido del producto y/o servicio hasta la entrega del mismo. La cadena de valor de los workflows abarca la innovación, las operaciones y el servicio post-venta.

La cuarta y última perspectiva del BSC es fundamental para conseguir las tres anteriores. Es la perspectiva de aprendizaje y crecimiento del personal. El BSC hace una referencia explícita a la importancia que tienen los Recursos Humanos en la consecución de los objetivos empresariales. La presente perspectiva incide en tres aspectos clave. Por un lado, se trata de potenciar las capacidades de los empleados, las ideas de mejora deben de provenir de los empleados que están cerca de los procesos y de los clientes. De otra manera, las organizaciones deben dotarse de sistemas de información respecto a los empleados en cuanto a su satisfacción, su retención y su desempeño. Los miembros de una organización deben sentirse motivados, deben tener libertad para tomar decisiones y actuar (empowerment), y deben tener una coherencia en sus objetivos. Para evaluar los parámetros del BSC construimos una escala denominada BSC-14 (Boada y Gil, 2007a).

La dirección estratégica de recursos humanos ha experimentado un gran avance en los últimos años Arthur, 1992; Brewster, 1993, 1995 y 1999; Cascio, 1989; Decenzo y Robbins, 1988; Delery y Doty, 1996; Delery y Shaw, 2001; Edgar y Geare, 2005; Huselid, 1995; MacDuffie, 1995; Milkovich y Boudreau, 1991; Papalexandris y Panayotopoulou, 2004; Pfeffer, 1994; Schuler, 1987; Schuler y Jackson, 1987a y 1987b; Sparrow y Hiltrop, 1994; Stavrou y Brewster, 2005; Tichy, Fombrun y Devanna, 1982; Wright y Snell, 1991). Algunas investigaciones relacionan la Gestión Estratégica de Recursos Humanos con el BSC pero de forma simplemente descriptiva (Hagood y Friedman, 2002; McPhail, Herington y Guilding, 2008; Rhodes, Walsh y Lok, 2008; Whitaker y Wilson, 2007).

En la presente investigación hemos contemplado las siguientes áreas de la Gestión Estratégica de Recursos Humanos, citadas por los autores anteriores, que detallamos a continuación: (1) Los "Valores y la Cultura" que deben ser conocidos por los empleados y deben propiciar su integración y su compromiso, así como la adaptación al entorno y la consecución de resultados por parte de la empresa, (2) la descripción y el análisis de todos los "Puestos de Trabajo" integrado en una Gestión de las Competencias, (3) la "Comunicación Interna" con el desarrollo de sistemas formales de aportación de ideas y sugerencias y de planes de comunicación organizacional, (4) la "Formación" que implica la elaboración, el seguimiento y la evaluación de un plan congruente con la estrategia de la organización, (5) la "Evaluación del Desempeño y del Rendimiento" por objetivos y por competencias, (6) la "Selección de Personal" que facilita el éxito de las nuevas incorporaciones y utiliza distintas técnicas como la entrevista y las pruebas de simulación, (7) la "Retribución Salarial" implica planes personalizados a las necesidades de los empleados, la identificación de su posición en el mercado retributivo y que la estructura salarial contemple la retribución salarial fija, variable y extrasalarial, (8) los "Procesos de Acogida y Desvinculación en la Empresa" que deben estar sujetos los empleados, (9) la "Planificación de Plantillas" a partir de un conjunto de previsiones internas (jubilaciones, rotación, nivel de producción, desarrollo de nuevos productos/servicios, conflictos colectivos, etc.) y externas (planes estratégicos y de marketing, evolución de la legislación, etc.), (10) el "Clima y la Motivación" que denota su influencia en el funcionamiento de la empresa, así como planes de acción para mejorar el clima laboral y la motivación, y la conciliación entre la vida laboral y la personal-familiar, (11) el "Trabajo de Equipo" que debe ser un compartido en la organización, permitiendo la creación de equipos de trabajo para solucionar problemas o para desarrollar oportunidades, (12) la "Gestión del Cambio" debe permitir que los empleados lo acepten y perciban los cambios como una oportunidad y un reto, así la organización debe facilitar los procesos de adaptación al cambio, (13) el "Liderazgo" que ha de facilitar el desarrollo de los empleados, favorecer los procesos de innovación y de delegación, y disponer de un margen importante de empowerment, así como modelo definido de competencias directivas, (14) las "Relaciones Laborales" que permite gestionar los conflictos de forma positiva y eficiente, existiendo una actitud proactiva de la empresa respecto a los representantes de los trabajadores y (15) el "Plan de Carrera" que posibilita tanto el desarrollo profesional interno para los empleados, como identificar el potencial o talento de las personas. Con el fin de poder diagnosticar la Gestión Estratégica de Recursos Humanos diseñamos la escala GERH-53 (Boada y Gil, 2007b).

Algunos estudios vinculan la relación de la Gestión Estratégica de Recursos Humanos con la gestión del conocimiento (Svetlik y Stavrou-Costea, 2007); con la innovación de las organizaciones (Cano y Cano, 2006); con la satisfacción (Garrido, Pérez y Antón, 2005); con la calidad del trabajo (De Prins y Henderickx, 2007); con el empowerment, la comunicación interna y la justicia interna (Tzafrir, Harel, 
Baruch y Dolan, 2004); con el rendimiento de los empleados (Ghebregiorgis y Karsten, 2007); con el rendimiento organizacional (Katou y Budhwar, 2006; Lin y Kuo, 2007; Harris, Cortvriend y Hyde, 2007; Hansson, 2007); con el cuidado de los pacientes hospitalizados (Kabene, Orchard, Howard, Soriano y Leduc, 2006); con los cambios estructurales (Morris y Farrell, 2007); con el liderazgo (Zacharatos, Hershcovis, Turner y Barling, 2007); con el desarrollo de las empresas del sector del automóvil (Schuler y Tarique, 2007) y con los aspectos transculturales (Aycan, AlHamadi, Davis y Budwar, 2007).

En este contexto, y atendiendo los planteamientos teóricos expuestos previamente, los objetivos de la presente investigación son: 1) Determinar si la Gestión Estratégica de Recursos Humanos (Variable Predictora) implementada en las empresas es pronosticadora de que los Procesos o Workflows (Variable Criterio) contemplados en el BSC tiendan a la excelencia; 2) Analizar si la Gestión Estratégica de Recursos Humanos (Variable Predictora) implantada en las organizaciones es inductora de que los clientes (Variable Criterio) tiendan a la satisfacción y a la fidelización (BSC); y 3)Verificar si la Gestión Estratégica de Recursos Humanos (Variable Predictora) diseñada en las empresas influye en los Resultados Financieros (BSC) (Variable Criterio) de la misma.

\section{Método}

\section{Participantes}

En el presente estudio participaron 222 directivos de organizaciones de la Comunidad Autónoma de Cataluña. La edad media de los mismos es de 36 años $(\mathrm{DT}=9.4)$. La antigüedad media en la empresa es de 8.3 años $(\mathrm{DT}=10.4)$ y la antigüedad media en el puesto es de 4.7 años $(\mathrm{DT}=4.8)$. La distribución de género es la siguiente: masculino (64.4\%) y femenino (35.6 1\%). La posición jerárquica de los entrevistados en la empresa es la que sigue: Director General o Gerente el $18.5 \%$, Director de RRHH el $17.6 \%$, otros responsables del Área de RRHH el 11.0\%, Directores de Área Funcional (No de RRHH) el $18.0 \%$ y otros Responsables de Área Funcional (No de RRHH) el $34.1 \%$. El nivel de titulación alcanzado por los encuestados se reparte del siguiente modo: el $31.5 \%$ son Diplomados o Ingenieros Técnicos; el $47.0 \%$ son Licenciados o Ingenieros Superiores; el $3.0 \%$ son Doctorados; y el $18.5 \%$ son otros sin especificar. En cuanto a la formación previa, la procedencia de sus estudios es: Ingeniería (16.0\%), Economía (23.7\%), Derecho (9.3\%), Psicología (8.8\%) y otros $(42.3 \%)$. En cuanto a las características de las empresas, la media de empleados de las mismas es de 1.123. Siendo la cota máxima de 134.000 empleados y la mínima 349. Respecto a los sectores socioeconómicos repre- sentados son los que siguen: El primario (agricultura, ganadería, pesca y minería) con un $9.80 \%$; el secundario (farmacéutica, química, metalúrgica, caucho y plástico, perfumería y cosmética, maquinaria y equipos, montaje, automóvil, maquinaria y electricidad) con el 45.29\%; el terciario (servicios destinados a la venta, seguros, comercio, ventas y agencias viaje) con el $34.31 \%$; y el cuaternario (telecomunicaciones, informática, internet, software y NN.TT) con el $10.60 \%$. En la muestra no ha habido presencia del sector público.

\section{Variables e Instrumentos}

A todos los directivos se les aplicó y se registraron los datos correspondientes a:

Datos Demográficos del Entrevistado: Edad, sexo, nivel de responsabilidad del entrevistado en la empresa, antigüedad (en el puesto y en la empresa), nivel de titulación alcanzado, ámbito de la formación previa, y formación especializada.

Datos Demográficos de la Empresa: Número total de empleados, número de empleados por niveles, sector económico donde se ubica la actividad, y la concentración o descentralización de las funciones de RR.HH.

Escala BSC-14 (Balanced Scorecard) (Boada y Gil, 2007a). Habiendo realizado una revisión de la literatura sobre el Balanced Scorecard de Kaplan y Norton, y no habiendo encontrado ningún cuestionario previo, se planteó la elaboración de un instrumento de 14 ítems que reflejase los tres parámetros de los cuatro que integra el modelo norteamericano. Los 14 ítems son valorados mediante una escala de Likert de cinco anclajes. Globalmente, la fiabilidad de la escala puede considerarse como muy razonable ( $\alpha$ de Cronbach $=.82$ ). El factor Finanzas incorpora 4 ítems, evalúa el rendimiento, la facturación y el beneficio de la empresa. Explica el $15.16 \%$ de la varianza total. Incluye ítems como por ejemplo. "El rendimiento de la organización sobre las inversiones es ...”. La fiabilidad es de .77. El factor Clientes tiene 4 ítems, incorpora aspectos que hacen referencia a la satisfacción, fidelización, cuota de mercado y estándares de calidad alcanzados (p.ej.,"La satisfacción de nuestros clientes es...”). Explica el $8.67 \%$ de la varianza total. El $\alpha$ de Cronbach es de .70. Y el factor Procesos o Workflows se explora con 6 ítems. Refleja tanto la eficiencia y planificación organizacional como la reducción de la burocracia interna y la potenciación de los niveles tecnológicos (por ej.," El desarrollo de nuevos productos y/o servicios es-...”). Es el que explica más varianza, el $31.53 \%$. Su índice de fiabilidad es de .77 .

Escala GERH-53 (Gestión Estratégica de Recursos Humanos) (Boada y Gil, 2007b). La presente escala consta de 53 ítems, a los cuáles se les realizó un Análisis Factorial Exploratorio, mediante la extracción 
de ejes principales y rotación oblimin. Se obtuvieron un conjunto de factores que integran las Políticas de Recursos Humanos. Los 53 ítems debían de ser puntuados a través de una escala de Likert de cinco posiciones. El coeficiente alfa de Cronbach para toda la escala alcanza un valor de .95 , puede decirse que presenta una muy adecuada consistencia interna. Los factores hallados fueron: 1) Valores y Cultura $(\alpha=.84)$ incluye ítems como "Los Valores de la organización están explicitados y definidos, y son conocidos por las personas", 2) Puesto de Trabajo $(\alpha=.76)$ incorpora ítems como "La organización tiene un documento con la Descripción y el Análisis de todos los Puestos”, 3) Comunicación Interna $(\alpha=.75)$, un ejemplo de ítem es "Existe un Plan de Comunicación Descendente", 4) Formación $(\alpha=.89)$ está constituído por ítems como "La empresa dispone de un Plan Anual de Formación acorde con el Plan Estratégico de la misma", 5) Evaluación del Desempeño y del Rendimiento ( $\alpha$ =.77), un ítem de referencia sería "La empresa tiene un sistema de evaluación del rendimiento por objetivos", 6) Selección de Personal $(\alpha=.70)$ contiene ítems como ? La entrevista es la prueba más significativa en el proceso de selección de mi empresa”, 7) Retribución Salarial $(\alpha=.70)$, un ítem representativo sería ?La empresa utiliza encuestas salariales para identificar su posición competitiva en el mercado retributivo"; 8) Acogida y Desvinculación de la empresa $(\alpha=70)$ incluye ítems como "Todas las nuevas incorporaciones están sujetas a un proceso formal de acogida e integración", 9) Planificación de Plantillas $(\alpha=.72)$ incorpora ítems como "En la organización se determina la plantilla necesaria según un conjunto de previsiones internas como jubilaciones, rotación, fugas, nivel de producción, desarrollo de nuevos productos / servicios, conflictos colectivos, etc.”, 10) Clima y Motivación $(\alpha=.70)$, un ejemplo de ítem es "En mi organización se realizan estudios de clima laboral", 11) Trabajo en Equipo $(\alpha=.80)$ está constituído por ítems como ?La organización fomenta la creación de equipos de trabajo para solucionar problemas o para desarrollar oportunidades", 12) Gestión del Cambio $(\alpha=.80)$, un ítem de referencia sería "La organización facilita los procesos de adaptación al cambio" 13) Liderazgo $(\alpha=.80)$ contiene ítems como ?Las personas disponen de un margen importante en la toma de decisiones (empowerment)", 14) Relaciones Laborales $(\alpha=.74)$, un ítem representativo sería "Los conflictos laborales son gestionados de forma positiva y eficiente" y 15) Plan de Carrera $(\alpha=.70)$ incluye ítems como "Existen programas de desarrollo profesional interno para los empleados".

\section{Procedimiento}

Se utilizó el muestreo aleatorio-accidental (Kerlinger, 2001) o también denominado no probabi- lístico de conveniencia (Gómez, 1990; Hernández, Fernández \& Baptista, 2000) tanto para los participantes como para las empresas. Los cuestionarios se entregaron de forma personalizada a los directivos que de manera voluntaria participaron en el presente estudio. Además, se garantizaba la absoluta confidencialidad en el tratamiento de los datos. A través de una entrevista, realizada por psicólogos-investigadores preparados expresamente para ello, se les explicó las instrucciones acerca de cómo rellenar los cuestionarios que se les entregaban. Durante la administración, había la presencia de al menos un psicólogo-investigador, quien garantizaba el tratamiento confidencial de la información y se ofrecía a resolver las dudas que pudieran surgir en la cumplimentación de las escalas. La recogida de datos se realizó dentro de las organizaciones, en horas laborables y en su puesto de trabajo.

\section{Análisis de datos}

La información obtenida tras la aplicación de los instrumentos de evaluación ha sido sometida a un análisis por medio del programa SPSS 15.0. Dado que nuestro objetivo ha sido aproximarnos a la varianza común asociada entre las variables predictoras (Gestión Estratégica de Recursos Humanos) y las variables criterio (Balanced Scorecard) se han realizado varios análisis de regresión lineal entre dos variables para comprobar su interacción. En este marco no presentamos regresiones con variables progresivamente aditivas de forma que las relaciones entre varias variables no queden desdibujadas por una interacción más compleja.

\section{Resultados}

En la Tabla 1 se describen las ecuaciones de regresión de la Gestión Estratégica de Recursos Humanos (Variables Predictoras, en adelante VP) sobre la variable Procesos o Workflows del BSC (Variables Criterio, en adelante VC). En dicha tabla se constata la aparición de quince significaciones $(p<0.01)$. Como puede observarse las Beta obtenidas son todas positivas. Así pues, se aprecia que todas las variables de la Gestión Estratégica de Recursos Humanos son predictoras de los Procesos o Workflows.

Respecto a la covarianza explicada $\left(\mathrm{r}^{2}\right)$ entre la Gestión Estratégica de Recursos Humanos y Procesos o Workflows, se puede observar que dos variables (1 y 13) sobrepasan el .300; ocho de ellas $(2,3,4,9,10,11$, 12 y 15$)$ se encuentran entre 200 y . 299; y cinco (5, $6,7,8$ y 14) entre .100 y .199. Las variables con más $\mathrm{r}^{2}$ son "1) Valores y Cultura" y "13) Liderazgo"; y con menos "5) Evaluación del Desempeño y Rendimiento." y "8) Acogida y Desv. Empresa". La $\mathrm{r}^{2}$ media de Procesos / Workflows es de .242. 
Tabla 1. Ecuación de regresión de las variables integrantes de la Gestión Estratégica de Recursos Humanos sobre la variable Procesos/ Workflows del CMI (Balanced Scorecard)

\begin{tabular}{lllr}
\hline V. Predictora & Beta & $\mathrm{r}^{2}$ & $\mathrm{t}$ \\
\hline 1) Valores y Cultura & .617 & .380 & 11.38 \\
2)Puestos de Trabajo & .471 & .221 & 7.63 \\
3) Comunicación Interna & .506 & .256 & 8.55 \\
4) Formación & .520 & .270 & 8.86 \\
5) Evaluación Desem y Rendim. & .368 & .136 & 5.76 \\
6) Selección de Personal & .422 & .178 & 6.67 \\
7) Retribución Salarial & .411 & .169 & 6.45 \\
8) Acogida y Desv. Empresa & .382 & .146 & 5.98 \\
9) Planificación Plantillas & .531 & .282 & 9.02 \\
10) Clima y Motivación & .545 & .297 & 9.37 \\
11) Trabajo de Equipo & .544 & .296 & 9.41 \\
12) Gestión del Cambio & .526 & .276 & 8.98 \\
13) Liderazgo & .574 & .329 & 10.00 \\
14) Relaciones Laborales & .406 & .165 & 6.25 \\
15) Plan de Carrera & .487 & .237 & 8.10
\end{tabular}

Nota. Variable Criterio = Procesos/Workflows. Todas las t de Student son significativas más allá de $\mathrm{p}<.001$

En la Tabla 2, las quince VP de la Gestión Estratégica de Recursos Humanos tienen un impacto considerable en la VC (Finanzas del BSC). En doce casos se observa la interrelación (siete $a p<0.01$, cinco a $p<0.05)$, en tres ocasiones no aparece ninguna correlación entre las variables (VP números: 8,11 y 14). En la presente tabla se indica que las Beta obtenidas son todas positivas. En este sentido, se estima que doce de las quince variables consideradas en el presente estudio son predictoras de las Finanzas del BSC. En cuan-

Tabla 2. Ecuación de regresión de las variables integrantes de la Gestión Estratégica de Recursos Humanos sobre la variable Finanzas del CMI (Balanced Scorecard)

\begin{tabular}{lcccc}
\hline V. Predictora & Beta & $\mathrm{r}^{2}$ & $\mathrm{t}$ & sig. \\
\hline 1) Valores y Cultura & .281 & .079 & 4.16 & .000 \\
2) Puestos de Trabajo & .211 & .045 & 3.02 & .003 \\
3) Comunicación Interna & .182 & .033 & 2.64 & .009 \\
4) Formación & .201 & .040 & 2.92 & .004 \\
5) Evaluación Desem y Rendim. .208 & .043 & 3.02 & .003 \\
6) Selección de Personal & .178 & .032 & 2.54 & .012 \\
7) Retribución Salarial & .179 & .032 & 2.57 & .011 \\
8) Acogida y Desv. Empresa & .112 & .013 & 1.60 & .111 \\
9) Planificación Plantillas & .182 & .033 & 2.60 & .010 \\
10) Clima y Motivación & .196 & .038 & 2.83 & .005 \\
11) Trabajo de Equipo & .120 & .014 & 1.72 & .087 \\
12) Gestión del Cambio & .164 & .027 & 2.36 & .019 \\
13) Liderazgo & .183 & .033 & 2.59 & .010 \\
14) Relaciones Laborales & .028 & .001 & 0.39 & .696 \\
15) Plan de Carrera & .280 & .078 & 4.14 & .000
\end{tabular}

Nota. Variable Criterio $=$ Finanzas to a la $\mathrm{r}^{2}$, se puede apreciar que todas las variables se encuentran en la cota 001 y .079. Las variables con más covarianza son "1) Valores y Cultura" y "15) Plan de Carrera" y con menos "14) Relaciones Laborales" y "8) Acogida y Desv. Empresa". La $\mathrm{r}^{2}$ media de Finanzas es de .036 .

Respecto a la Tabla 3, se presentan las regresiones de la VP (Gestión Estratégica de Recursos Humanos) sobre la VC (Clientes del BSC). Se aprecia que todas la VP tienen una muy buena predicción sobre VC, así aparecen quince significaciones todas a $p<0.01$.

De esta forma, se constata que las quince variables consideradas en el presente estudio son predictoras de la variable Clientes del BSC. También se muestra que las Beta obtenidas son todas positivas.

En cuanto a la covarianza explicada se puede observar que una variable está entre .200 y .299; diez están entre .100 y .199; y cuatro se sitúan entre .000 y .099 . Las variables con más $\mathrm{r}^{2}$ son " 1 ) Valores y Cultura" y "13) Liderazgo"; y las menores "7) Retribución Salarial” y "5) Evaluación Desempeño y Rendimiento". La ${ }^{2}$ media de Clientes es de .139.

Tabla 3. Ecuación de regresión de las variables integrantes de la Gestión Estratégica de Recursos Humanos sobre la variable Clientes del CMI (Balanced Scorecard).

\begin{tabular}{llll}
\hline V. Predictora & Beta & $\mathrm{r}^{2}$ & $\mathrm{t}$ \\
\hline 1) Valores y Cultura & .506 & .256 & 8.49 \\
2) Puestos de Trabajo & .320 & .103 & 4.80 \\
3) Comunicación Interna & .389 & .151 & 6.12 \\
4) Formación & .411 & .169 & 6.54 \\
5) Evaluación Desem y Rendim. & .243 & .059 & 3.62 \\
6) Selección de Personal & .301 & .090 & 4.50 \\
7) Retribución Salarial & .273 & .074 & 4.05 \\
8) Acogida y Desv. Empresa & .309 & .096 & 4.69 \\
9) Planificación Plantillas & .388 & .151 & 6.03 \\
10) Clima y Motivación & .387 & .150 & 6.02 \\
11) Trabajo de Equipo & .419 & .176 & 6.66 \\
12) Gestión del Cambio & .418 & .174 & 6.64 \\
13) Liderazgo & .432 & .187 & 6.79 \\
14) Relaciones Laborales & .338 & .114 & 5.05 \\
15) Plan de Carrera & .368 & .135 & 5.71 \\
\hline
\end{tabular}

Nota. Variable Criterio $=$ Clientes. Todas las $\mathrm{t}$ de Student son significativas más allá de $\mathrm{p}<.001$

Finalmente, en la Tabla 4 se presentan los quince Coeficientes de Determinación $\left(\mathrm{r}^{2}\right)$ de las variables de la Gestión Estratégica de Recursos Humanos, ordenados según la $\mathrm{r}^{2}$ media de mayor a menor, teniendo en cuenta los tres parámetros (Procesos / Workflows, Finanzas y Clientes) del BSC. También hemos obtenido la $\mathrm{r}^{2}$ media para cada uno de los indicadores. 
Tabla 4. Coeficientes de determinación $\left(\mathrm{r}^{2}\right)$, ordenados según la $\mathrm{r}^{2}$ media de mayor a menor, entre las variables integrantes de la Gestión Estratégica de Recursos Humanos y las variables Procesos / Workflows, Finanzas y Clientes del CMI (Balanced Scorecard)

\begin{tabular}{lcccc}
\hline & $\begin{array}{c}\text { V. Criterio } \\
\text { Procesos }\end{array}$ & $\begin{array}{c}\text { V. Criterio } \\
\text { Finanzas }\end{array}$ & $\begin{array}{c}\text { V. Criterio } \\
\text { Clientes }\end{array}$ \\
\hline V. Predictora & $\mathrm{r}^{2}$ & $\mathrm{r}^{2}$ & $\mathrm{r}^{2}$ & $r^{2}$ media \\
\hline 1) Valores y Cultura & .380 & .079 & .256 & .238 \\
13) Liderazgo & .329 & .033 & .187 & .183 \\
11) Trabajo de Equipo & .296 & .014 & .176 & .162 \\
10) Clima y Motivación & .297 & .038 & .150 & .161 \\
12) Gestión del Cambio & .276 & .027 & .174 & .159 \\
4) Formación & .270 & .040 & .169 & .159 \\
9) Planificación Plantillas & .282 & .033 & .151 & .155 \\
15) Plan de Carrera & .237 & .078 & .135 & .150 \\
3) Comunicación Interna & .256 & .033 & .151 & .146 \\
2) Puestos de Trabajo & .221 & .045 & .103 & .123 \\
6) Selección de Personal & .178 & .032 & .090 & .100 \\
14) Relaciones Laborales & .165 & .001 & .114 & .093 \\
7) Retribución Salarial & .169 & .032 & .074 & .091 \\
8) Acogida y Desv. Empresa & .146 & .013 & .096 & .085 \\
5) Evaluación Desem y Rendim. & .136 & .043 & .059 & .079 \\
& .242 & .036 & .139 & \\
\hline \multicolumn{1}{r}{$r^{2}$ media } & .246 & & &
\end{tabular}

\section{Discusión y Conclusiones}

En la presente investigación tratamos de determinar la relación existente entre un conjunto de variables derivadas de la Gestión Estratégica de Recursos Humanos y los tres componentes esenciales del BSC. Los datos obtenidos muestran evidencia empírica que existe una relación entre las variables que hemos propuesto, aunque con ciertas matizaciones que trataremos de explicar.

Los resultados obtenidos en este estudio empírico no pueden ser comparados ya que no hemos encontrado estudios equivalentes al efectuado por nosotros, si nos atenemos tanto al marco teórico como a la metodología utilizada y los análisis estadísticos realizados. Algunos de ellos, que presentamos a continuación, abordan la presente temática de forma meramente descriptiva y no analizan las correlaciones entre ningún tipo de variables. Uno de ellos, el publicado por Hagood y Friedman (2002), se efectuó en una institución pública norteamericana y contempla un conjunto de índices como la satisfacción del cliente, el tiempo utilizado en los procesos internos, la congruencia entre las habilidades y las necesidades de aprendizaje de los empleados. Otro, se efectuó en una entidad bancaria norteamericana y tiene en consideración variables como el rendimiento, la retribución y la identificación del talento (Whitaker y Wilson, 2007). El tercero (Rhodes, Walsh y Lok, 2008) se desarrolló en una entidad bancaria de Indonesia y examina aspectos como la cultura, el liderazgo y las prácticas de Recursos
Humanos. Y cuarto, McPhail, Herington y Guilding (2008) se desarrolló en el sector hotelero $(\mathrm{N}=14$, managers de RRHH) y focaliza la investigación en uno de los cuatro ámbitos del BSC, el "aprendizaje y crecimiento".

Puesto que todas las variables de la Gestión Estratégica de Recursos Humanos son predictoras $(p<0.01)$ del ámbito Procesos / Workflows del BSC (Kaplan y Norton, 2006 y 2007), se confirma el primer objetivo para todas las quince variables consideradas, así la Gestión Estratégica de Recursos Humanos (VP) implementada en las empresas es pronosticadora de que sus Procesos o Workflows (VC) tiendan a la excelencia. En consecuencia, podemos considerar la importancia, en gran medida, de un conjunto de aspectos. Por un lado, cuando la cultura y los valores de la empresa son compartidos por las personas que trabajan en ella se potenciarán tanto los estándares de calidad alcanzados por la compañía como la planificación interna en la organización. Probablemente, si el estilo de liderazgo de los directivos facilita el desarrollo y el crecimiento de los colaboradores y, además, la formación se contempla como un elemento fundamental de crecimiento para los empleados todo ello facilitará el desarrollo de nuevos productos y/o de nuevos servicios. En otro sentido, si existe un sistema formal de aportación de ideas y sugerencias con el fin de recoger las soluciones creativas e innovadoras que proponen los empleados tendrá una clara incidencia en la eficiencia de cómo se resuelven los problemas organizativos. También, la potenciación del talento de las personas y la elaboración por parte de las empresas de un modelo propio de gestión por competencias facilitará unos adecuados niveles tecnológicos y los sistemas de información implícitos en éllos. Así pues, la Gestión Estratégica de Recursos Humanos contribuye al desarrollo de la cadena de valor implícita en los Procesos que va desde la detección de las necesidades del cliente hasta la satisfacción de las mismas. Efectivamente, inciden en el proceso de innovación (identificación del mercado y creación - oferta del producto y/o servicio), en el proceso operativo (construcción y entrega de los productos y/o servicios) y en el proceso de servicio post-venta (mantenimiento y derivados).

En cuanto al segundo objetivo de nuestro estudio que consiste en verificar si la Gestión Estratégica de Recursos Humanos diseñada en las organizaciones influye en los Resultados Financieros de la misma, se aprecia que doce de las quince variables integrantes de la Gestión Estratégica de Recursos Humanos son predictoras (siete a $p<0.01$ y cinco a $p<0.05$ ) del ámbito Finanzas del (Kaplan y Norton, 2006 y 2007). De este modo, podemos considerar que si la cultura organizacional propicia tanto la adaptación al entorno socioeconómico de la misma como el desarrollo profesional de los empleados probablemente el rendimiento de la organización será positivo y eficiente. También, parece probable que la organización de los puestos de trabajo 
y, explícitamente, la descripción de los mismos facilitará la obtención de rendimientos organizacionales. Asimismo, si la compañía tiene un sistema de evaluación del rendimiento de los empleados en una modalidad de "evaluación por objetivos" los excedentes empresariales se verán favorecidos positivamente. En el mismo sentido, se constata que si la organización dispone de planes de formación anuales para sus empleados se facilitará la obtención de buenos resultados. Así, dichas políticas permitirán potenciar cada una de las fases del ciclo de vida de una organización que van desde un crecimiento paulatino de la cuota de mercado hasta la sostenibilidad del mismo. Así mismo, aquellas políticas pueden permitir a la compañía una adaptación a las nuevas exigencias financieras de derivadas de la situación coyuntural del mercado.

Finalmente, el tercer objetivo trata de analizar si la Gestión Estratégica de Recursos Humanos implantada en las organizaciones es inductora de que los clientes tienden a la satisfacción y a la fidelización, se estima que las quince variables que integran la Gestión Estratégica de Recursos Humanos son predictoras $(p<0.01)$ del ámbito Clientes del BSC (Kaplan y Norton, 2006 y 2007). De esta forma, podemos estimar que cuando los valores culturales de la organización que potencien el aprender de los clientes están explicitados y son conocidos por los empleados es probable que la organización conserve y, quizás, aumente su cuota de mercado en el sector que está desarrollando su actividad. Por otro lado, una óptima comunicación ascendente y descendente a nivel organizacional entre los empleados y los departamentos es posible que incida en una óptima resolución de quejas de los clientes. También, podemos considerar que si la empresa va a diseñar un plan de formación que contemple acciones formativas encaminadas a la atención al cliente es probable que los clientes de la organización sean fidelizados con más intensidad. Asimismo, si el trabajo en equipo es un valor compartido en toda la organización en el sentido de asumir responsabilidades ante el cliente es plausible que la satisfacción de los clientes aumente. Además, cuando la organización facilita los procesos de adaptación al cambio de los empleados, cuando éstos aceptan el cambio y los mismos empleados perciben los cambios como una oportunidad y un reto es entonces cuando se facilitará la adquisición de nuevos clientes. De este modo, la Gestión Estratégica de Recursos Humanos incidirá en el incremento de los clientes, la retención y fidelización de los mismos y especialmente la satisfacción de los clientes; contribuyendo todo ello al aumento de la cuota de mercado, a la imagen y el prestigio.

Para dar una mayor claridad a los resultados obtenidos podemos indicar que habiendo realizado la media de los tres coeficientes de determinación $\left(\mathrm{r}^{2}\right)$ obtenidos de cada una de las variables que constituyen la Gestión Estratégica de Recursos Humanos, si dichos coeficientes los ordenamos de mayor a menor podemos determinar tres grupos. El primer grupo, que denominamos Alfa, estaría compuesto por cuatro de ellas y su coeficiente de determinación medio oscilaría entre .238 y .161. Consideramos que cuatro variables de la Gestión Estratégica de Recursos Humanos (1) Valores y Cultura, 13) Liderazgo, 11) Trabajo en Equipo y 10) Clima y Motivación) serían las que más influyen en la optimización de los parámetros de la organización (Finanzas, Clientes y Procesos) propuestos por el BSC (Kaplan y Norton, 2006 y 2007). El segundo grupo, nominado como Beta, estaría integrado por siete variables de la Gestión Estratégica de Recursos Humanos (12) Gestión del cambio, 4) Formación, 9) Planificación de Plantillas, 15) Plan de Carrera, 3) Comunicación Interna, 2) Puestos de Trabajo y 6) Selección de personal) las cuales tienen una influencia intermedia en la obtención de unos índices positivos del BSC (Kaplan y Norton, 2006 y 2007). El coeficiente de determinación medio oscilaría entre .159 y .123. Y el tercer grupo, llamado Omega, sería el que tendría menos influencia en los indicadores del BSC propuestos por Kaplan y Norton (2006 y 2007). El coeficiente de determinación medio oscilaría entre .093 y .079 . Este grupo estaría integrado por cuatro variables de la Gestión Estratégica de Recursos Humanos (14) Relaciones Laborales, 7) Retribución Salarial, 8) Acogida y Desvinculación en la Empresa y 5) Evaluación del Desempeño y del Rendimiento).

A la luz de los resultados, podemos indicar algunas conclusiones. En primer lugar, se han cumplido los tres objetivos propuestos en la presente investigación dado que las políticas de Recursos Humanos son predictoras de los tres parámetros analizados del BSC. Una segunda cuestión importante es que la variable "1) Valores y Cultura" es la variable de la Gestión Estratégica de Recursos Humanos que es más pronosticadora de todas las consideradas. En tercer lugar, como hemos visto anteriormente no todas las variables de la Gestión Estratégica de Recursos Humanos tienen la misma importancia, en este sentido hay un grupo configurado por cuatro de ellas que aportan más valor añadido. Cuarta, se aprecia que las variables de la Gestión Estratégica de Recursos Humanos son más predictoras en el ámbito de los Procesos $\left(\mathrm{r}^{2}\right.$ media $\left.=.242\right)$ y de los Clientes $\left(\mathrm{r}^{2}\right.$ media $\left.=.139\right)$ que en el de las Finanzas $\left(\mathrm{r}^{2}\right.$ media $=.036)$. La evidencia de los resultados nos indica que las empresas deberían insistir en potenciar las políticas alfa dado que las hace más sostenibles. Y quinta, respecto a los participantes se observa que el $70 \%$ de los directivos encuestados no están vinculados al Área de Recursos Humanos, sin embargo éstos asocian la efectividad de la Gestión Estratégica de Recursos Humanos a los resultados del BSC.

En cuanto a la aplicabilidad del presente estudio empírico podemos indicar alguna de ellas sin la pretensión de ser exhaustivos. Primera, vistos los resultados es de suma importancia que los responsables del área de Recursos Humanos se vinculen cada vez más a los 
sistemas de gestión encaminados a evaluar los resultados de las organizaciones a través del BSC. En segundo lugar, puesto que hay elementos de la Gestión Estratégica de Recursos Humanos que contribuyen a aumentar de forma significativa la cadena de valor de los diferentes ámbitos de la organización se deriva que no pueden ser sujetas a externalización (outsourcing) y, además, deben ser potenciadas por la dirección de la organización. Tercera, observamos que los aspectos intangibles de la organización, en este caso la Gestión Estratégica de Recursos Humanos, cada vez más van a ocupar un rol estratégico dentro de la empresa, siendo imprescindible que los directivos y empleados aboquen su gestión en el crecimiento y la formación de las personas. Un cuarto aspecto, la Gestión Estratégica de Recursos Humanos es necesaria no tan solo para la creación de una estrategia sostenible para la organización sino para que los empleados y directivos puedan desarrollarse tanto personal como profesionalmente. Quinta, se hace necesario priorizar la Gestión Estratégica de Recursos Humanos, así se deberá realizar un esfuerzo por conocer cuáles son aquellos aspectos que ayudan más al crecimiento de los empleados y, también, los que están más alineados con la estrategia de la propia organización. Y sexta, deben crearse siner- gias entre los distintos elementos que integran la Gestión Estratégica de Recursos Humanos con el fin de que entre ellas no se produzcan contradicciones y, a la vez, se interfieran negativamente; en este sentido, saber interrelacionar las diferentes políticas y mantener una coherencia entre ellas nos aportará información para mejorar los propios procedimientos internos, potenciando la formación y las capacidades de los equipos de colaboradores.

En cuanto a los ámbitos de futuro derivados de la presente investigación, pueden ser tres. Por un lado, consideramos que deberían de comprobarse la consistencia de los resultados obtenidos tanto en empresas de menor tamaño, por ejemplo las PYMEs y las empresas familiares, como en el ámbito de la Administración Pública. Un segundo ámbito a considerar sería la necesidad de tener en cuenta las diferencias entre los distintos sectores socioeconómicos (primario, secundario, terciario y cuaternario). Y finalmente, sería oportuno establecer la relación entre la Gestión Estratégica de Recursos Humanos con aspectos que, hasta el día de hoy según la literatura revisada, no han sido motivo de investigación como los riesgos psicosociales, la motivación, el compromiso, el absentismo y la rotación de los empleados.

\section{Extended Summary}

In the 1990s, Kaplan and Norton (1992, 1996, 2001, 2005a, 2005b, 2006, 2007) introduced the Balanced Scorecard (BSC) model, allowing organisations to be planned and evaluated. This model considers four strategic perspectives of organisations. The first is the financial perspective, which allows returns that are higher than the investment made to be obtained. The second is the customer perspective, for which modern organisations need to identify market segments and existing and potential customers. The third is the internal process (or workflow) perspective of the organisation, a process which has to be improved and tend towards excellence. The fourth is the learning and growth perspective for employees, which explicitly refers to the importance of Human Resources in attaining business objectives. Strategic Human Resources Management has progressed tremendously in recent years. Some studies (Hagood and Friedman, 2002; McPhail, Herington and Guilding, 2008; Rhodes, Walsh and Lok, 2008; Whitaker and Wilson, 2007) link Strategic Human Resources Management to the BSC, though only descriptively.

In this study we have taken account of a number of areas of Strategic Human Resources Management as cited by the aforementioned authors: values and culture, the description and analysis of all jobs, internal communication, training, appraisal of carrying out and performance, recruitment and selection, pay, the company's induction and exit processes, workforce plan- ning, work climate and motivation, teamwork, change management, leadership, industrial relations and career plans.

In this context, and taking account of the theoretical approaches mentioned earlier, the objectives of this study are: 1. To ascertain whether or not Strategic Human Resources Management (Predictive Variable) implemented in companies predicts that Processes or Workflows (Criterion Variable) considered in the BSC tend towards excellence; 2 . To analyse whether or not Strategic Human Resources Management (Predictive Variable) implemented in organisations makes Customers (Criterion Variable) tend towards satisfaction and loyalty (BSC); and 3. To check whether or not Strategic Human Resources Management (Predictive Variable) designed in companies impacts on their Financial results (BSC) (Criterion Variable).

\section{Method}

\section{Participants}

Two hundred and twenty-two directors from organizations in the Autonomous Community of Catalonia took part in the study. The mean age of the cohort was 36 ( $\mathrm{SD}=9.4)$. The mean length of service in the company was 8.3 years $(\mathrm{SD}=10.4)$ and the mean length of service in the job was 4.7 years $(\mathrm{SD}=4.8)$. The distri- 
bution by sex was as follows: male (64.4\%) and female $(35.61 \%)$. The hierarchical position of the interviewees in their respective companies was as follows: Managing Directors or General Managers (18.5\%); Human Resources Directors (17.6\%); Other Human Resources Managers (11.7\%); Functional Area Directors (not Human Resources) (18\%); and Other Functional Area Managers (not Human Resources) $(34.1 \%)$. In terms of education, their qualifications were in: Engineering (16\%); Economics (23.7\%); Law (9.3\%); Psychology (8.8\%); and Others (42.3\%). Regarding economic sectors represented, these were as follows: primary $(9.8 \%)$; secondary $(45.29 \%)$; tertiary $(34.31 \%)$; and quaternary $(10.6 \%)$. There was no presence of the public sector in the sample.

\section{Measures}

Data were applied to and recorded for all the directors in relation to:

Demographic Data of the Interviewee: Age, sex, level of responsibility of the interviewee in the company, length of service (in the job and in the company), qualifications, educational field and specialist training.

Demographic Data of the Company: Total number of employees, number of employees by grade, economic sector business activity, concentration or decentralisation of Human Resources functions.

BSC-14 scale (Balanced Scorecard) (Boada and Gil, 2007a). Having reviewed the literature on Kaplan and Norton's Balanced Scorecard and not having found any previous questionnaire, the creation of a 14-item instrument was considered in order to reflect three of the four parameters contained in the American model. The 14 items are evaluated using a five-point Likert scale. Overall, the reliability of the scale can be considered to be very reasonable (Cronbach's Alpha $=0.82$ ).

SHRM-53 scale (Strategic Human Resources Management) (Boada and Gil, 2007b). This scale contains 53 items, which were subjected to Exploratory Factor Analysis through principal axis extraction and oblimin rotation. A set of factors contained in Human Resources Policies was obtained. The 53 items had to be scored using a five-point Likert scale. The Cronbach's Alpha coefficient for the whole scale reaches a value of 0.95 , so it can be asserted that its internal consistency is very good. The factors found were: 1) Values and Culture (Alpha =0.84); 2) Job $($ Alpha $=0.76) ; 3)$ Internal Communication $($ Alpha $=$ 0.75); 4) Training (Alpha $=0.89)$; 5) Appraisal of Carrying out and Performance (Alpha $=0.77$ ); 6) Recruitment and Selection (Alpha = 0.70); 7) Pay (Alpha $=0.70) ; 8)$-Induction and Exit Processes $($ Alpha $=0.70) ; 9)$-Workforce Planning $($ Alpha $=$ 0.72); 10. -Climate and Motivation (Alpha =0.70); 11) Teamwork (Alpha $=0.80) ; 12$ ) Change Management $($ Alpha $=0.80) ; 13)$ Leadership $($ Alpha $=0.80) ; 14)$ -
Industrial Relations $($ Alpha $=0.74)$; and 15) Career Plan (Alpha $=0.70)$.

\section{Procedure}

The study used random accidental sampling (Kerlinger, 2001), also known as nonprobability convenience sampling (Gómez, 1990; Hernández, Fernández and Baptista, 2000), for participants and companies alike.

\section{Results}

Table 1 shows the regression equations of Strategic Human Resources Management (Predictive Variables or PVs) over the Process or Workflow variable of the BSC (Criterion Variables or CVs). This table also shows 15 statistically significant results $(p<0.01)$. Therefore, it is possible to see that Strategic Human Resources Variables are predictive of Process or Workflow. In Table 2, the fifteen Strategic Human Resources Management PVs have a significant impact on the CV (BSC Financial). In 12 instances there is a correlation (seven are $p<0.01$ and five are $p<0.05$ ) and in three instances there is no correlation between the variables (PV numbers 8,11 and 14). Table 3 shows the regressions of the PV (Strategic Human Resources Management) over the CV (BSC Customer). It is possible to see that all the PVs have a good VC predictive power, hence the 15 statistically significant results, all of which are $p<0.01$. Thus, it is found that the 15 variables considered in this study are predictive of the BSC Customer variable. Finally, Table 4 shows the 15 Coefficients of Determination $\left(\mathrm{r}^{2}\right)$ of the Strategic Human Resources Management variables, sorted in descending order (highest to lowest) by mean $\mathrm{r}^{2}$, taking account of the three BSC parameters (Process/ Workflow, Financial and Customer). We have also obtained the mean $\mathrm{r}^{2}$ for each of the indicators.

\section{Discussion}

In this study we endeavoured to ascertain the relationship between a set of Strategic Human Resources Management variables and the three essential components of the BSC. The data obtained provide empirical evidence that a relationship between the variables that we have put forward does indeed exist although some clarification is required, which we shall attempt to explain.

Given that all the Strategic Human Resources Management variables are predictive $(p<0.01)$ of the Process/Workflow perspective of the BSC (Kaplan and Norton, 2006 and 2007), the first objective for all 15 variables considered is confirmed. Thus, Strategic 
Human Resources Management (PV) implemented in companies predicts that Processes or Workflows (CV) tend towards excellence. Consequently, we are able to consider the importance of a set of aspects. Firstly, when the culture and values of a company are shared by the people working for it, both the company's quality standards and the organisation's internal planning will be strengthened. Obviously, if the directors' leadership style facilitates employee growth and development and if, in addition, training is considered to be a fundamental component of employee growth, all of that will assist the development of new products and/or new services. Secondly, if a formal system is in place for employees to provide ideas and make suggestions with the aim of gathering creative, innovative solutions, it will have a clear impact on the way in which organisational problems are resolved. Therefore, Strategic Human Resources Management contributes to the development of the value chain implicit in Processes, ranging from the detection of customers' needs to the satisfaction of those needs. Furthermore, it has an impact on the innovation process (market identification and creation/offer of products and/or services), on the operational process (construction and delivery of products and/or services) and on the after sales service process (maintenance and derivatives).

Regarding the second objective of our study, which consists in checking whether or not Strategic Human Resources Management designed in organisations impacts on their Financial results, it is possible to see that 12 of the 15 variables contained in Strategic Human Resources Management are predictive (seven are $\mathrm{p}<0.01$ and five are $p<0.05$ ) of the Financial perspective of the BSC (Kaplan and Norton, 2006 and 2007). Thus, we are able to consider that if the organisational culture favours its adaptation to the socioeconomic environment on the one hand, and employee professional development on the other, it is likely that the organisation's performance will be positive and efficient. It is likely that the organisation of jobs and, explicitly, their description will facilitate organisational performance. Similarly, if the company has a system of appraising employee performance on the basis of "appraisal by objectives", there will be a positive effect on business gains.

Finally, the third objective endeavours to analyse whether or not Strategic Human Resources Management implemented in organisations makes customers tend towards satisfaction and loyalty, and it is considered that the 15 variables contained in Strategic Human Resources Management are predictive $(p<0.01)$ of the Customer perspective of the BSC (Kaplan and Norton, 2006 and 2007). Thus, we are able to consider that when an organisation's cultural values aimed at strengthening customer learning are explicit and employees are aware of them, it is likely that the organisation will maintain and possibly increase its market share in the sector in which it undertakes its business activities. Furthermore, we are able to consider that if a company designs a training plan that includes training actions aimed at customer care, it is likely that the organisation's customers will become more loyal. Moreover, when an organisation facilitates processes for its employees to adapt to change, and when these processes are accepted and the employees themselves perceive the changes as an opportunity and a challenge that is when new customers will be secured. Thus, Strategic Human Resources Management will impact on the number of customers, causing them to rise, and on customer retention and loyalty; all of which contributes to an increase in market share, image and reputation.

In the light of these results, we are able to point towards a number of conclusions. First, the three objectives put forward in this study have been accomplished, given that Human Resources policies are predictive of the three BSC parameters analysed. A second very important issue is that the variable " 1 . Values and Culture" is the most predictive Strategic Human Resources Management variable of all those considered. Third, as already seen earlier, not all the Strategic Human Resources Management variables have the same importance and, in this sense, there is a group of four variables that contribute more added value. Fourth, it is possible to see that the Strategic Human Resources Management variables are more predictive for Process and Customer perspectives than for the Financial perspective.

\section{References}

Arthur, J. B. (1992). The linkbetween business strategy and industrial relations systems in American steel minimills. Industrial and Labor Relations Review, 45, 488-506.

Aycan, Z., Al-Hamadi, A.B., Davis, A. y Budhwar, P. (2007). Cultural orientations and preferences for HRM policies and practices: the case of Oman. International Journal of Human Resource Management, 18, 11-32.

Boada, J. y Gil, C. (2007a). BSC-14: Análisis de las propiedades psicométricas y estructura factorial. Tarragona, España: Universitat Rovira i Virgili. No Publicado.

Boada, J. y Gil, C. (2007b). Estudio de la bondad psicométrica y estructura factorial de una escala de la Gestión Estratégica de Recursos Humanos (GERH-53). Tarragona, España: Universitat Rovira i Virgili. No Publicado.

Braam, G, J., Benders, J. y Heusinkveld, S. (2007). The balanced scorecard in the Netherlands - An analysis of its evolution using print-media indicators. Journal of Organizational Change Management, 20, 866-879.

Brewster, C. (1993). Developing a 'European' model of Human Resource Management. International Journal of Human Resource Management, 4, 765-784.

Brewster, C. (1995). Towards a European Model of Human Resource Management. Journal of International Business 
Studies, First Quarter, 1-2.

Brewster, C. (1999). Strategic Human Resource Management: the value of different paradigms. Management International Review, 39, 45-64.

Cano, C.P. y Cano, P.Q. (2006). Human resources management and its impact on innovation performance in companies. International Journal of Technology Management, 35, 11-28.

Cascio, W. F. (1989). Managing human resources: productivity, quality of work life, profits. New York, USA: McGraw Hill.

Decenzo, D.S. y Robbins, S.P. (1988). Personnel / human resource management. Englewood Cliffs, USA: Prentice Hall.

Delery, J. E. y Doty, D. H. (1996). Modes of theorizing in strategic Human Resource Management: Test of universalistic, contingency, and configurational performance predictions. The Academy of Management Journal, 39, $802-835$.

Delery, J. E. y Shaw, J. D. (2001). The strategic management of people in work organizations: Review, synthesis, and extension. Research in Personnel and Human Resource Management, 20, 165-197.

De Prins, P. y Henderickx, E. (2007). HRM effectiveness in older people's and nursing homes: The search for best (quality) practices. Nonprofit and Voluntary Sector Quarterly, 36, 549-571.

Dessler, G. (1988). Personnel Management. Englewood Cliffs, USA: Prentice Hall.

Edgar, F. y Geare, A. (2005). HRM practice and employe attitudes: diferent measures-different results. Personnel Review, 34, 534-549.

Garrido, M.J., Perez, P. y Anton, C. (2005). Determinants of sales manager job satisfaction. An analysis of Spanish industrial firms. International Journal of Human Resource Management, 16, 1934-1954.

Ghebregiorgis, F. y Karsten, L. (2007). Employee reactions to human resource management and performance in a developing country. Personnel Review, 36, 722-738.

Gómez, J. (1990). Metodología de encuesta por muestreo. En J. Arnau, M.T. Anguera y J. Gómez. (Cooed.). Metodología de la Investigación en Ciencias del Comportamiento (pp.239-310). Murcia: Universidad de Murcia.

Greatbanks, R. y Tapp, D. (2007). The impact of balanced scorecards in public sector environment-Empirical evidence from Dunedin City Council, New Zealand. International Journal of Operations \& Production Management, 27, 846-873.

Hagood, W.O. y Friedman, L. (2002). Using the Balanced Scorecard to measure the performance of your HR Information System. Public Personnel Management, 31, 543-557.

Hansson, B. (2007). Company-based determinants of training and the impact of training on company performance Results from an international HRM survey. Personnel Review, 36, 311-331.

Harris, C., Cortvriend, P. y Hyde, P. (2007). Human resource management and performance in healthcare organisations. Journal Health Organizational Management, 21, 448-459.

Hernández, R., Fernández, C. y Baptista, P. (2000). Metodología de la investigación. México: McGraw-Hill.

Huselid, M. (1995). The impact of human resource management practices on turnover, productivity,and corporate financial performance. Academy of Management Journal, 38, 635-672.

Kabene, S.M., Orchard, C., Howard, J.M., Soriano, M.A. y Leduc, R. (2006). The importance of human resources management in health care: a global context. Human Resource Health, 4, 200-220.

Kaplan, R. S. y Norton, D.P (1992). The Balanced Scorecard - Measures that Driver Performance. Harvard Business Review, 70, 71-79.

Kaplan, R. S. y Norton, D.P. (1996). The Balanced Scorecard: Translating strategy into action. Harvard. USA: Harvard Bussiness School Press.

Kaplan, R. S. y Norton, D.P. (2001). Cómo utilizar el Cuadro de Mando Integral. Para implantar y gestionar su estrategia. Barcelona, España: Gestión 2000.

Kaplan, R .S. y Norton, D.P. (2005a). The balanced scorecard: Measures that drive performance. Harvard Business Review, 83, 172-181.

Kaplan, R. S. y Norton, D.P. (2005b). The office of strategy management. Harvard Business Review, 83, 72-84

Kaplan, R.S. y Norton, D.P. (2006). How to implement a new strategy without disrupting your organization. Harvard Business Review, 84, 100-109.

Kaplan, R.S. y Norton, D.P. (2007). Using the balanced scorecard as a strategic management system.Harvard Business Review, 85, 150-158.

Katou, A. A. y Budhwar, P.S. (2006). Human resource management systems and organizational performance: A test of a mediating model in the Greek manufacturing context. International Journal of Human Resource Management, 17, 1223-1253.

Keramati, A., Mehrabi, H. y Mousakhani, M. (2008). The role of human, organization and technology in building competitive advantage with CRM: The case of iranian internet service industry. 6th IEEE International Conference on Industrial Informatics, 1-3, 1212-1217.

Kerlinger, F.N. (2001). Investigación del comportamiento: Métodos de investigación en ciencias sociales. México: McGraw-Hill.

Klein, D.N. y Nicholson, I.R. (2006). Costs of predoctoral clinical psychology internship training in a Canadian health care setting. Canadian Psychology/Psychologie Canadienne, 47, 333-342.

Lin, C.Y. y Kuo T.H. (2007). The mediate effect of learning and knowledge on organizational performance. Industrial Management \& Data Systems, 107, 1066-1083.

MacDuffie, J. P. (1995). Human resource bundles and manufacturing performance: Organizational logic and flexible production systems in the world auto industry. Industrial and Labor Relations Review, 48, 197-221.

McPhail, R., Herington, C. y Guilding, C. (2008). Human 
resource managers' perceptions of the applications and merit of the balanced scorecard in hotels. International Journal of Hospitality Management, 27, 623-631.

Michalska, J. (2005). The usage of The Balanced Scorecard for the estimation of the enterprise's effectiveness. Journal of Materials Processing Technology, 162, 751758 .

Milkovich, G. T y Boudreau, J. W. (1991). Human Resource Management. Homewood: Irwin.

Morris, J. y Farrell, C. (2007). The 'post-bureaucratic' public sector organization. New organizational forms and HRM in ten UK public sector organizations. International Journal of Human Resource Management, 18, 1575-1588.

Papalexandris, N. y Panayotopoulou, L. (2004). Exploring the mutual interaction of societal culture and human resource management practices. Employee Relations, 26 , 495-509.

Park, E.J. y Huber, D.L. (2007). Balanced scorecards for performance management. Journal of Nursing Administration, 37, 14-20.

Pfeffer, J. (1994). Competitive advantage through people. Boston: Harvard Business School Press.

Rampersad, H.K. (2008). The way to a high-performance culture with the Total Performance Scorecard. Strategic Change, 17, 43-55.

Rhodes, J., Walsh, P. y Lok, P. (2008). Convergence ad divergence issues in strategic management-Indonesia's experience with the Balanced Scorecard in HR management. The International Journal of Human Resource Management, 19, 1170-1185.

Schreiber, W. (2007). Balanced Scorecard: A Management Tool for Psychiatric Hospitals. Psychiatrische Praxis, 34, 157-159.

Schuler, R.S. (1987). Personnel and Human Resource Management Choices and Organizational Strategy. Human Resource Planning, 10, 1- 17.

Schuler, R.S. y Jackson, S.E. (1987a). Linking Competitive Strategies with Human Resource Management Practices. The Academy of Management Executive, 1, 207-219.
Schuler, R.S. y Jackson, S.E. (1987b).Organizational Strategy and Organization Level as Determinants of Human Resource Management Practices. Human Resource Planning, 10, 125-141.

Schuler, R.S. y Tarique, I. (2007). International human resource management: a North American perspective, a thematic update and suggestions for future research. International Journal of Human Resource Management, 18, 717-744.

Sparrow, P. y Hiltrop, J. M. (1994). European Human Resource Management in Transition. Londres: PrenticeHall.

Stavrou, E. y Brewster, C. (2005). The configurational approach to linking strategic human resource management bundles with business performance: Myth or reality? Management Review, 16, 186-201.

Svetlik, I. y Stavrou-Costea, E. (2007). Connecting human resources management and knowledge management. International Journal of Manpower, 28, 197-206.

Tichy, N., Fombrun, C. y Devanna, M. (1982). Strategic Human Resource Management. Sloan Management Review, 23, 47-61.

Tzafrir, S.S., Harel, G.H., Baruch, Y. y Dolan, S.L. (2004). The consequences of emerging HRM practices for employees' trust in their managers. Personnel Review, 33, 628-647.

Whitaker, D. y Wilson, L. (2007). Human Capital measurement: From insight to action. Organization Development Journal, 25, 59-64.

Wisniewski, M. y Olafsson, S. (2004). Developing balanced scorecards in local authorities: A comparison of experience. International Journal of Productivity and Performance Management, 53, 602-610.

Wright, P.M. y Snell, S.A. (1991). Toward an integrative view of strategic human resource management. Human Resource Management Review, 1, 203-225.

Zacharatos, A., Hershcovis, M.S., Turner, N. y Barling J. (2007). Human resource management in the North American automotive industry - A meta-analytic review. Personnel Review, 36, 231-254.

Manuscrito Recibido: 12/3/2009

Revisión Recibida: 6/7/2009

Aceptado: 13/7/2009 